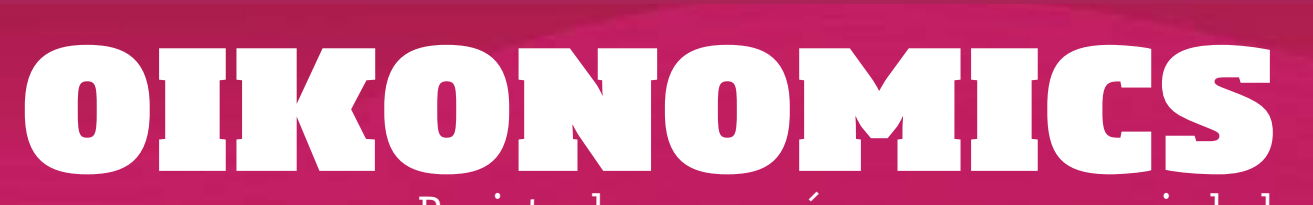

Revista de economía, empresa y sociedad

OPTIMIZACIÓN DE LA CADENA DE SUMINISTRO

\title{
Ancho ferroviario y logística 4.0 en el Corredor Mediterráneo
}

\section{Domingo Pérez Mira}

Profesor colaborador de los Estudios de Economía y Empresa (UOC)

RESUMEN El Corredor Mediterráneo se concibe como una doble vía ferroviaria de alta velocidad que discurrirá desde la frontera francesa hasta Algeciras, uniendo ciudades tan importantes como Barcelona, Valencia, Alicante, Murcia y Málaga y conectándolas a su vez con el resto de Europa. Sin embargo, para su puesta en marcha es necesario el desarrollo de tecnologías de cambio de ancho para las plataformas ferroviarias, que permitan la transición entre los diferentes anchos de vía europeos que dificultan el tránsito de mercancías por ferrocarril.

A su vez, en términos empresariales, la logística 4.0 está emergiendo con fuerza, proporcionando nuevas herramientas digitales que modifican tanto las operaciones como los procesos de negocio. Se trata de una transformación completa e integral, basada en la digitalización de la información a lo largo de toda la cadena de suministro, desde las fases iniciales hasta la llegada del producto final al cliente, integrando además la logística inversa.

La convergencia de la tecnología ferroviaria de ejes de ancho variable para el transporte de mercancías con la logística 4.0 en los procesos de SCM (supply chain management) permitirá incrementar la productividad y la competitividad empresarial a escala internacional.

PALABRAS CLAVE logística 4.0; Corredor Mediterráneo; gestión cadena de suministro; SCM; transformación digital; ancho variable; transporte mercancías 


\title{
Rail track gauge and logistics 4.0 in the Mediterranean Corridor
}

\begin{abstract}
The Mediterranean Corridor is a double high-speed railway that will run from the French border to Algeciras, joining cities as important as Barcelona, Valencia, Alicante, Murcia and Malaga, and connecting them in turn with the rest of Europe. However, it is necessary to develop gauge change technologies for railway platforms, since, in Europe, there are several track gauges that hinder the transit of goods by rail.

Logistics 4.0 modifies business operations and business processes to incorporate new tools and digital uses. It is a complete and integral transformation process, based on the digitization of information throughout the whole process, from the initial phases right through to the arrival of the end product to the customer, as well as integrating reverse logistics.

The convergence of variable-width axes rail technology for freight transport with Logistics 4.0 in SCM (Supply Chain Management) processes will allow increasing productivity and business competitiveness at an international level.
\end{abstract}

KEYWORDS logistics 4.0; Mediterranean Corridor; supply chain management; SCM, digital transformation; axis gauge; freight transportation

\section{Introducción}

España es uno de los países de la UE con menor porcentaje de transporte de mercancías por ferrocarril. Apenas un $5 \%$ de lo que se transporta se hace por tren, frente al 23,3 \% de Alemania o el 17,4 \% de Francia. El desarrollo del Corredor Mediterráneo, que discurre desde la frontera francesa hasta Algeciras, permitirá desarrollar la economía española al conectarla con el resto del continente, integrándola en el transporte europeo de mercancías con todas las ventajas que supone el ferrocarril.

En paralelo, la sociedad se encuentra inmersa en plena «transformación digital». Los procesos de negocio evolucionan gracias a las nuevas tecnologías, lo que genera cambios constantes en el entorno competitivo. No se trata de mejorar ni optimizar los métodos tradicionales, sino de modificar las operaciones y procesos de negocio para incorporar las nuevas herramientas y usos digitales, que permiten además nuevos tipos de innovación y creatividad.

En este marco, la emergencia de nuevas herramientas de big data y business inteligence, propias de la logística 4.0, implican una manera distinta de pensar y 
actuar en el ámbito global que afecta, inherentemente, a tres pilares básicos de los procesos logísticos de la organización: experiencia de cliente, procesos y modelo de negocio.

La aplicación de este nuevo modelo de gestión operacional mediante las ventajas de la tecnología de material rodante de ancho variable y la logística 4.0 en la SCM permite automatizar procesos, monitorizar y mejorar la visibilidad de las operaciones, incrementar la productividad, flexibilizar las operaciones y reducir los costes operativos.

\section{El Corredor Mediterráneo}

El Corredor Mediterráneo consistirá en una doble vía ferroviaria de alta velocidad que discurrirá desde la frontera francesa hasta Algeciras, uniendo ciudades tan importantes del litoral como Barcelona, Valencia, Alicante, Murcia y Málaga, y conectándolas a su vez con el resto de Europa.

Figura 1. Corredor Mediterráneo

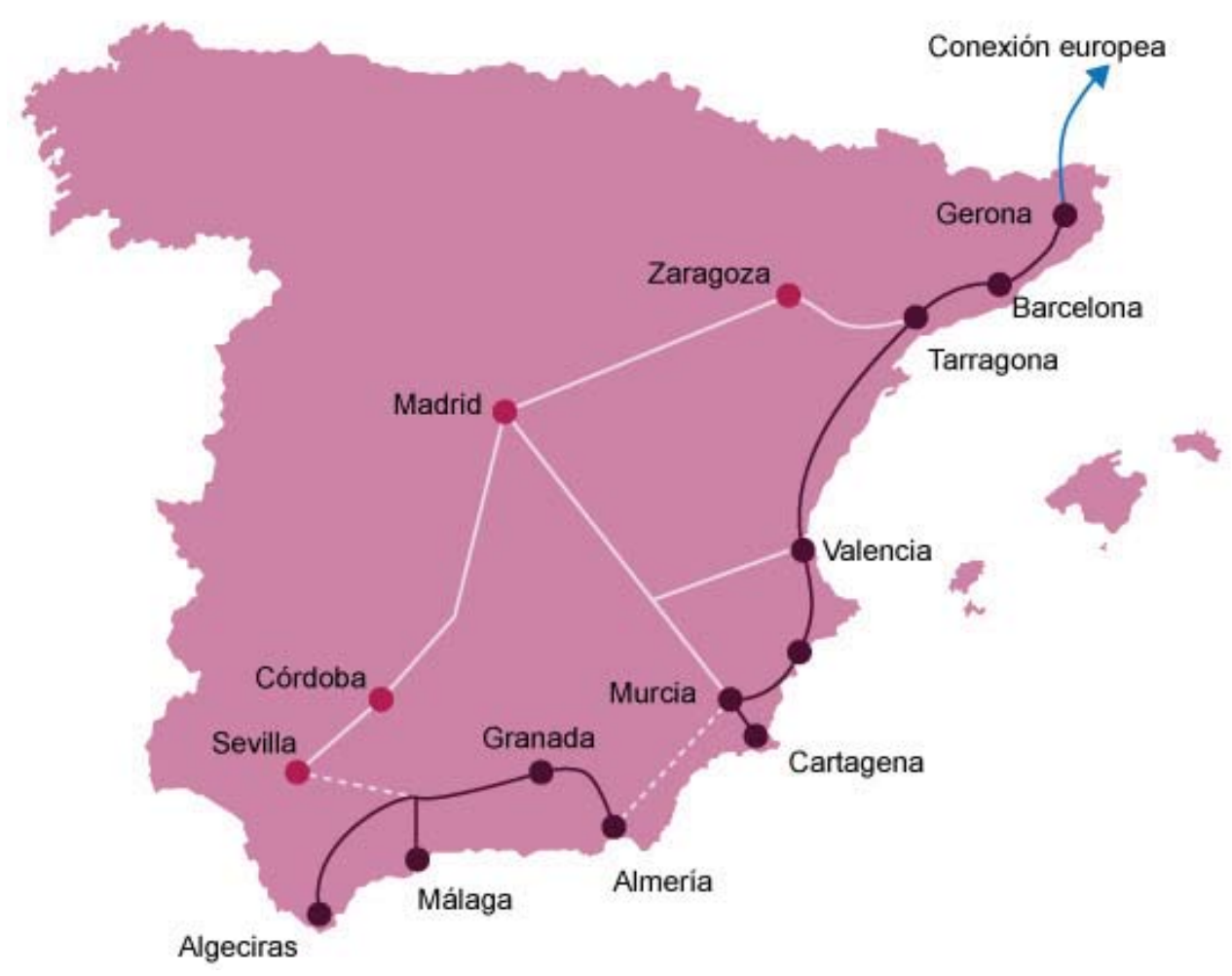

El Corredor Mediterráneo se encuentra dentro del «Gran Eje FERRMED», cuya red comprende la zona de mayor actividad económica y logística de la UE, incluyendo puertos y aeropuertos. Incorpora las áreas que van desde San Petersburgo, en Ru- 
sia, pasando por el sur de Finlandia, Estonia, la mayor parte de Letonia, y la parte occidental de Lituania, la ciudad de Kaliningrado, en Rusia, la mitad sur de Suecia, el área de la ciudad de Oslo en Noruega, Dinamarca, la mayor parte de Alemania, una fracción del noroeste de Polonia, los Países Bajos, Bélgica, Luxemburgo, el sector suroriental de Gran Bretaña, la mayor parte de Francia, Suiza, Liechtenstein, la esquina occidental de Austria, el noroeste de Italia, Andorra, la parte este y meridional de España (Corredor Mediterráneo) y, finalmente, el norte de Marruecos y de Argelia.

Esta red transeuropea, a la que se unirá el Corredor Mediterráneo, vertebrará Europa de norte a sur. 3.500 kilómetros que conectarán el 54 \% de los habitantes y el 66 \% del producto interior bruto de la Unión Europea, impulsando la competitividad de nuestro continente.

Sin embargo, en Europa existen varios anchos de vía, lo que dificulta el tránsito de mercancías por ferrocarril. De hecho, el eje FERRMED discurre por una gran variedad de países; así, se producen cambios de ancho en las fronteras de la Unión Europea con Rusia y en la de España con Francia.

Históricamente, el ancho de vía característico de la red ferroviaria española y portuguesa es de 1.668 milímetros (ancho ibérico) y, desde la decisión adoptada en 1988 de construir las nuevas líneas españolas de alta velocidad con ancho internacional de vía, de 1.435 milímetros (UIC, siglas del francés Union Internationale des Chemins de Fer), hace que haya coexistencia de diferentes anchos de vía tanto dentro de la red ferroviaria de España como en las fronteras con Francia. En el resto de Europa (Francia, Alemania, etc.) y en China, por ejemplo, la vía es de ancho internacional.

\section{Tecnología ferroviaria de ancho variable para mercancías}

España es referente europeo en la investigación tecnológica y consecución real de sistemas de cambio de ancho ferroviario. El número de cambiadores actualmente en operación es elevado; crecen de forma permanente y permiten el aprovechamiento de redes de ferrocarril convencionales para alta velocidad. Tras la inclusión del Corredor Mediterráneo en la Red Básica Transeuropea de Transporte, se ha hecho más fehaciente la necesidad urgente de adaptación al ancho europeo. Para ello, las soluciones propuestas han ido desde la sustitución total del ancho ibérico al UIC, en los tramos dedicados únicamente al transporte de viajeros, así como la implantación de un tercer carril en toda la longitud del Corredor.

Tradicionalmente, el tráfico de mercancías transfronterizo entre España y Europa, a través de Francia, ha requerido solucionar el problema de cambio de ancho ya que ha generado frecuentes problemas de explotación, además de operaciones 
de coste logístico elevado. Para superar esta barrera, se desarrollaron sistemas de intercambio de ejes y de vagones, creándose una serie de instalaciones posicionadas de forma estratégica para llevar a cabo este tipo de operaciones. De esta forma, para el paso de una red a otra, se cambian las plataformas, o los ejes de las mismas, lo cual presenta dos inconvenientes:

- El proceso de cambio de ejes es lento (se requiere al menos 10 minutos por eje).

- El coste es elevado. Necesita el empleo de mano de obra especializada y de instalaciones específicas que deben estar redundadas, para no convertir la frontera en un cuello de botella, en caso de un elevado número de vagones.

El diseño de un sistema alternativo de ejes, específico para plataformas ferroviarias de mercancías, compatible con los vagones actuales y que abarque otros anchos como el ruso (1.520 mm), se postula como una solución clave para impulsar la competitividad del ferrocarril en el transporte de mercancías, tanto en el ámbito nacional como internacional.

Actualmente, la tecnología ferroviaria permite que los trenes de pasajeros puedan circular por vías de distintos anchos, pero este reto no está consolidado para el caso de trenes de mercancías, donde las plataformas ferroviarias deben poder soportar una carga de hasta 22,5 Tm por eje. ADIF, el Administrador de Infraestructuras Ferroviarias, está desarrollando y homologando conjuntamente con la iniciativa privada ejes de ancho variable y cambiadores de ancho para el transporte de mercancías.

Básicamente, el sistema de ancho variable permite que los ejes de los vagones puedan adaptarse de forma automática para circular por anchos de vía diferentes. Esta adaptación se produce a baja velocidad, sin necesidad de detener la marcha, cuando el tren atraviesa un punto de la vía en el que debe estar instalado un dispositivo cambiador de ancho específico. Los vagones entran con un ancho de vía y salen con otro.

Técnicamente, el sistema Eje OGI está compuesto por un conjunto de ejes y ruedas desplazables, unidos mediante un sistema de cerrojo que, como principal característica, forma un conjunto sólido sin partes móviles durante el uso normal, sin necesidad de rodamientos o elementos adicionales. Se basa en un elemento plano de biela-manivela, que gira solidario al propio eje para asegurar el movimiento axial del mecanismo que bloquea cada rueda en la posición adecuada a cada ancho de vía.

Esta tecnología permite, además, reemplazar directamente los ejes convencionales de vagones y vehículos ferroviarios existentes, facilitando así su actualización para aprovechamiento futuro en conexiones ferroviarias con vías de distinto ancho. 


\section{Logística 4.0}

Más allá de la transformación infraestructural, cabe entender esta innovación en un contexto de cambio acelerado en el seno empresarial. La logística 4.0 ha emergido con fuerza, y con ella nuevas herramientas tecnológicas que pueden revolucionar el modo de gestionar los negocios. Atendiendo al transporte ferroviario, son especialmente relevantes las siguientes herramientas de análisis de datos:

- Cloud computing: infraestructura, plataformas y aplicaciones en modelo servicio. Permite nuevas capacidades de adaptación y escalabilidad. Alta flexibilidad para necesidades de almacenamiento o esfuerzo computacional para grandes y pequeñas empresas.

- Big data / inteligencia artificial: uso de gran cantidad de datos y reconocimiento de patrones de comportamiento de cara a la definición de indicadores de proceso y apoyo a la toma de decisión.

- Conectividad: todo conectado e internet de las cosas (IoT), que permiten nuevas formas de interacción entre personas y máquinas.

- Movilidad: modifica el modo como las personas interaccionan, consumen información y desarrollan trabajo colaborativo.

La mejora de la interconexión completa y en tiempo real, así como la autonomía en la logística, son los retos que afrontan cada vez más empresas para optimizar sus operaciones. La logística 4.0 es un proceso de transformación completo e integral basado en la digitalización de la información a lo largo de toda la cadena de suministro, desde las fases iniciales hasta la llegada del producto final al cliente, integrando además la logística inversa. Se trata de modificar las operaciones y procesos de negocio para incorporar las nuevas herramientas y usos digitales.

La conectividad y colaboración entre todos los agentes de la cadena son dos fundamentos básicos que, mediante la apertura de nuevas interfaces y de nuevas programaciones, permitirá garantizar una producción más personalizada, gestión de envíos más eficiente en función de la previsión de la demanda, reducción de stocks y tiempo de almacenaje, optimización de rutas, geolocalización de clientes, etc. Igualmente, con la creciente tecnología blockchain, la logística 4.0 permitirá automatizar procesos de compras (e-procurement), contratos de suministros o garantizar la trazabilidad de productos.

Todas están aplicaciones derivan en la máxima eficiencia operacional, y serán susceptibles de generar nuevos datos a través del tratamiento con la tecnología big data y la inteligencia de negocio. Podrán generar nuevo conocimiento y nuevas aplicaciones, que facilitarán la interconexión entre todos los servicios y actores de 
la cadena de suministro, realimentando al sistema sucesiva y constantemente para seguir optimizándolo mediante tecnología de machine learning.

Como ejemplo, la logística 4.0 aplicada a contenedores de mercancías permite que estos informen automáticamente sobre su propio estado, posición, contenidos y destino. Además, al tratar cada elemento contenedor como una entidad inteligente independiente, estos podrán comunicarse con los transportistas e incluso con otros contenedores para lograr optimizar el flujo logístico.

La antigua logística utilizaba volúmenes grandes de mercancías estándar para buscar un descenso de los costes, lo que generaba ineficiencias tanto en costes financieros como de manutención y almacenamiento de productos. La logística 4.0 también permite, en último término, optimizar los medios de pago. Se trata, en resumen, de una mejora en agilidad, eficacia y eficiencia en todo el proceso logístico.

\section{Optimización de la gestión de la cadena de suministro (SCM)}

Standler (2002) define la SCM (supply chain manegement) como «la tarea de integrar diferentes organizaciones a lo largo de toda la cadena coordinando el flujo de materiales y de información de forma que satisfaga la demanda de los clientes incrementando la competitividad de toda la cadena».

Al hablar de SCM, se renuncia a la gestión aislada de las empresas y, por tanto, al planteamiento de objetivos individuales como única vía para conseguir los resultados esperados. El profesor estadounidense John F. Nash (premio Nobel de Economía en 1994) realizaba la siguiente afirmación en su tesis económica: «Un sistema económico alcanza su máxima eficiencia cuando cada uno de los elementos que lo forman buscan su máxima eficiencia, al tiempo que la del conjunto del sistema».

Por tanto, la SCM abarca desde los proveedores de materias primas hasta el cliente final, pasando por todos los procesos intermedios de producción, almacenaje, preparación de pedidos, distribución y comercialización. Esto implica la coexistencia de dos flujos fundamentales en la cadena de suministro, que además son bidireccionales, ya que van de proveedor a cliente, pasando por todos los procesos intermedios, y de cliente a proveedor. Estos flujos son: materiales, de información y de conocimiento.

En este ámbito, la convergencia en la optimización de los flujos de materiales por ferrocarril aplicando la tecnología de ejes de ancho variable, y la optimización de los flujos de información y conocimiento mediante la logística 4.0, en una situación estratégica como es el Corredor Mediterráneo, implica directamente el incremento de la productividad y la competitividad empresarial a escala internacio- 
nal. Esta convergencia tecnológica permitirá automatizar procesos, monitorizar y mejorar la visibilidad de las operaciones, incrementar la productividad, flexibilizar las operaciones y reducir los costes operativos.

\section{Conclusiones}

El desarrollo del Corredor Mediterráneo, conjuntamente con la tecnología de eje ancho variable para el transporte de mercancías por ferrocarril, permitirá a las empresas españolas disponer de un medio rápido, ágil y económico, tanto para el transporte de sus materias primas procedentes de sus proveedores como para el suministro de los productos finales a sus clientes.

La implantación de la logística 4.0 en las empresas debe plantearse como una estrategia para reformular el modelo de negocio, del que dependen los procesos para materializar su visión empresarial, las personas que soportan los procesos de gestión del negocio y los sistemas, que son las herramientas que constituyen su soporte tecnológico.

Incorporar en los planes estratégicos la logística 4.0, dentro de los procesos de SCM, conjuntamente con el desarrollo del Corredor Mediterráneo, permitirá acelerar la economía española incrementando su productividad y competitividad, facilitando además el poder acceder en mejores condiciones al mercado internacional.

Es un desafío diseñar e implantar adecuadamente los planes de transformación en las empresas definiendo la ruta adecuada en cada caso. Se requiere generar actitudes positivas hacia la digitalización y cultura de gestión del cambio en las que las personas son clave para garantizar su correcta implantación.

\section{Referencias bibliográficas}

BERROZPE, A. (2012). La cadena de valor de los operadores logísticos en España: un análisis empírico. Madrid: Lulu.

BOWERSOX, D.; CLOSS, D.; BIXBY, M. (2007). Administración y logística en la cadena de suministro. Madrid: McGraw Hill.

CARBONELL, F.; PARÍS, J. (2017). «El proceso de construcción del arco mediterráneo: una calle de doble dirección». Papers: Regió Metropolitana de Barcelona: territori, estratègies i planejament, 2007: N. ${ }^{\circ} 44$, Planificación de infraestructuras y territorio. El arco mediterráneo.

CASANOVAS, A.; Cuatrecasas, LI. (2003). Logística empresarial. Barcelona: Gestión 2000.

COMPANYS, R. (2005). Diseño de sistemas productivos y logísticos. EPSEB-UPC. 
GARCÍA, A. (2007, enero-febrero). «Cambiadores de ancho, trenes de ancho variable y tercer carril: Nuevas soluciones a un viejo problema». Anales de mecánica y electricidad.

LAUDON, K.; LAUDON, J. (2004). Sistemas de información gerencial: administración de la empresa digital. Madrid: Pearson Educación.

OBSERVATORIO E-COMMERCE. «Informe sobre tendencias en la logística e-commerce para el 2014».

PÉREZ-LARA, M.; SAUCEDO-MARTÍNEZ, J.; SALAIS-FIERRO, T. (2016). «Caracterización de modelo de negocio en el marco de industria 4.0». Congreso Internacional de Logística y cadena de suministro, CiLOG2016.

PITA, A. (2006). Infraestructuras ferroviarias. Barcelona: Edicions UPC.

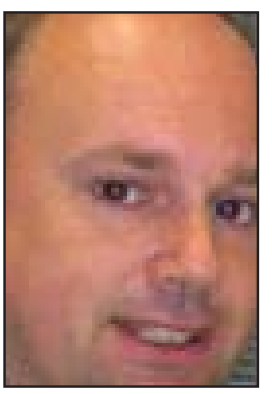

\section{Domingo Pérez Mira dperezmi@uoc.edu Profesor colaborador de los Estudios de Economía y Empresa (UOC)}

Doctor ingeniero industrial. Director de investigación y desarrollo en proyectos de colaboración entre universidad y empresa. Máster en Transformación Digital. Experto en logística y operaciones para el diseño y gestión de proyectos estratégicos en el incremento de la productividad y competitividad empresarial. Cuenta con gran experiencia en proyectos, tanto en el ámbito industrial como en el de infraestructura de obra civil y de edificación, con especialización en diferentes áreas de negocio: construcción, concesiones, industrial, transporte ferroviario y edificación.

Los textos publicados en esta revista están sujetas -salvo que se indique el contrario- a una licencia de Reconocimiento 3.0 España de Creative Commons. Podéis copiarlos, distribuirlos, comunicarlos públicamente y hacer obras derivadas siempre que reconozcáis los créditos de las obras (autoría, nombre de la revista, institución editora) de la manera especificada por los autores o por la revista. La licencia completa se puede consultar en http://creativecommons.org/licenses/by/3.0/es/deed.ca.

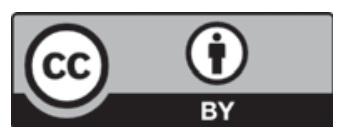

